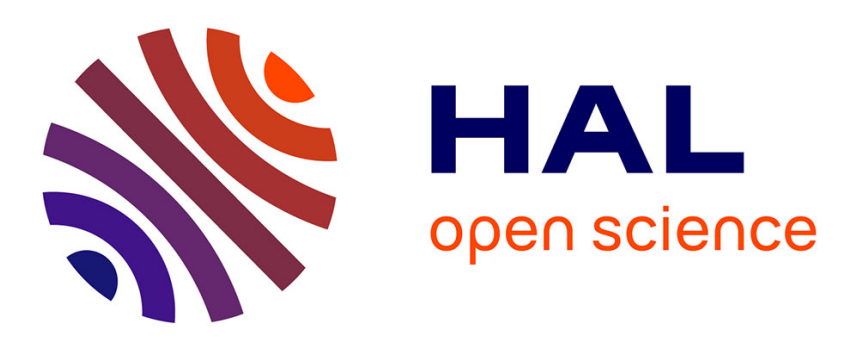

\title{
Global sensitivity analysis of analytical vibroacoustic transmission models
}

Jean-Loup Christen, Mohamed Ichchou, Bernard Troclet, Olivier Bareille, Morvan Ouisse

\section{- To cite this version:}

Jean-Loup Christen, Mohamed Ichchou, Bernard Troclet, Olivier Bareille, Morvan Ouisse. Global sensitivity analysis of analytical vibroacoustic transmission models. Journal of Sound and Vibration, 2016, 368, pp.121-124. 10.1016/j.jsv.2016.01.009 . hal-01480125

\section{HAL Id: hal-01480125 \\ https://hal.science/hal-01480125}

Submitted on 1 Mar 2017

HAL is a multi-disciplinary open access archive for the deposit and dissemination of scientific research documents, whether they are published or not. The documents may come from teaching and research institutions in France or abroad, or from public or private research centers.
L'archive ouverte pluridisciplinaire HAL, est destinée au dépôt et à la diffusion de documents scientifiques de niveau recherche, publiés ou non, émanant des établissements d'enseignement et de recherche français ou étrangers, des laboratoires publics ou privés. 


\title{
Global sensitivity analysis of analytical vibroacoustic transmission models
}

\author{
Jean-Loup Christen ${ }^{\mathrm{a}}$, Mohamed Ichchou ${ }^{\mathrm{a}}$, Bernard Troclet ${ }^{\mathrm{b}, \mathrm{c}}$, Olivier \\ Bareille $^{\mathrm{a}}$, Morvan Ouisse ${ }^{\mathrm{d}}$ \\ ${ }^{a}$ École Centrale de Lyon, Université de Lyon, 36 avenue Guy de Collongue, 69130 \\ Écully, France \\ ${ }^{b}$ Airbus Defence and Space, 66 Route de Verneuil, 78133 Les Mureaux Cedex, France \\ ${ }^{c}$ LMT, ENS Cachan, Université Paris-Saclay, 61 Avenue du Président Wilson, 94230 \\ Cachan \\ ${ }^{d}$ FEMTO-ST Applied Mechanics, ENSMM / UBFC, UMR CNRS 6174, 24 chemin de \\ l'Épitaphe, 25000 Besançon
}

\begin{abstract}
Noise reduction issues arise in many engineering problems. One typical vibroacoustic problem is the transmission loss (TL) optimisation and control. The TL depends mainly on the mechanical parameters of the considered media. At early stages of the design, such parameters are not well known. Decision making tools are therefore needed to tackle this issue. In this paper, we consider the use of the Fourier Amplitude Sensitivity Test (FAST) for the analysis of the impact of mechanical parameters on features of interest. FAST is implemented with several structural configurations. FAST method is used to estimate the relative influence of the model parameters while assuming some uncertainty or variability on their values. The method offers a way to synthesize the results of a multiparametric analysis with large variability. Results are presented for transmission loss of isotropic, orthotropic and sandwich plates excited by a diffuse field on one side. Qualitative trends found agree with the physical expectation. Design rules can then be set up
\end{abstract}


for vibroacoustic indicators. The case of a sandwich plate is taken as an example of the use of this method inside an optimisation process and for uncertainty quantification.

Keywords: FAST, sound transmission loss, composite structures, diffuse field

\section{Introduction}

Noise control can be a very important topic in engineering, and noise reduction problems can take many forms. Among these, noise transmission is about the transmission of energy from an incident sound field through a structure into an enclosure. Typical situations include for example the phonic isolation of a building in civil engineering, noise reduction inside a plane's cabin or a car. One of the most used indicators in vibroacoustics is the transmission loss (TL), which is the ratio of incident to transmitted energies, expressed in decibels. Transmission loss through composite structures depends on numerous structural parameters, such as Young's modulus, density, honeycomb geometry or damping ratio of each constituent. Noise transmission through isotropic plates has become a classical vibroacoustic problem, for which analytical solutions are derived in textbooks $[1,2]$. The case of orthotropic plates has been studied by Guyader and Lesueur [3]. Renji et al. proposed models for the transmission loss of sandwich panels [4]. All these models are based on analytical expressions of bending wavenumbers in the considered plate. At early design stages, these parameters are unknown with potentially wide variation ranges, due to either uncertainty or design latitude. In order to efficiently set up an optimisation problem, it can be of 
first importance to find which parameters contribute most to TL variability. Later on in the design cycle, lack of knowledge will be reduced, but uncertainty may remain on some parameters, due for example to identification issues or industrial tolerances [5]. It is therefore important to find out how parameter variability will affect vibroacoustic indicators. Identifying which parameters contribute most to output variability can help focus on them for optimisation or uncertainty reduction.

A number of methods have been developed to address uncertainties in vibroacoustics. Reynders [6] uses the maximum entropy principle to compute confidence intervals for the TL of walls between two uncertain rooms, where the probability densities and cross-correlations of the considered parameters are known. Batko and Pawlik [7] use a method derived from interval arithmetics to estimate variability intervals for the same kind of constructions. Regarding industrial structures, a stochastic boundary element method (BEM) has been proposed by D'Amico et al. [8, 9] to estimate the variability of noise radiation by structures with geometric uncertainties. Cicirello and Langley [10] combined parametric and non-parametric uncertainty analyses on a hybrid FE-SEA method. In their recent book, Ohayon and Soize [11] review methods of uncertainty assessment in vibroacoustics, including nonparametric uncertainties. All these approaches give interval estimates of the model output given input parameter uncertainty, but do not describe the influence of each parameter on this variability.

Sensitivity analysis aims at identifying the relative influence of parameters. One of the most used methods is to study the partial derivatives of the model output with respect to all variables, which gives local estimates 
around a reference point. Global sensitivity analysis (GSA) [12] on the other hand aims at deriving indicators of influence for broad variation ranges. The ANOVA class of methods uses the variance decomposition [13] as an estimate for the sensitivity of each parameter. The computation of each term in the variance decomposition requires time-consuming calculation. The Fourier amplitude sensitivity test (FAST) was developed by Cukier et al. [14] to reduce the computation time, with application in the study of complex chemical reactions. This method has later been reused by Iooss et al. [15] for radiologic risk assessment models. Ouisse et al. [16] applied the FAST method to porous material models, regarding acoustic impedance and absorption. This work was later extended to different models of porous materials with focus on microgeometry in [17]. We propose here an application of the FAST method to the analytical models of the transmission loss of infinite flat plates to illustrate the potential of global sensitivity analysis in this field.

It is important to remark that the ranking of parameters by order of influence on the transmission loss is not absolute, but depends on frequency. As the frequency ranges considered for noise transmission may be quite wide, the system will present very different behaviours in different regions of the excitation spectrum. Transmission of noise through a plate is due to the excitation of travelling waves in the plate by the incident sound, and the radiation on the other side created by these travelling waves. Radiation efficiency [18] varies depending on the relative wavenumbers of the waves propagating in the structure and the velocity of sound in the fluid. The peak of transmission is around the coincidence frequency, where these wavenumbers match. Below this frequency it is well known (see [1] for example) that 
the mass of the plate will be dominant, while for a plane wave, the bending stiffness will be preponderant in the higher frequency range. For anisotropic constructions, these results are still valid and well established [3], but the coincidence frequency is no longer well defined.

The main claims of the paper are: a study of the sensitivity of transmission loss of isotropic plates under plane wave and diffuse field excitations to parametric uncertainties. The trends provided by the FAST method are compared with common qualitative trends in the vibroacoustic community. The case of an orthotropic thin plate is then studied in the same way. Finer features corresponding to that case are highlighted that could not be obtained as easily with asymptotic expansion. Finally a less common sandwich panel is studied in the same framework, in order to investigate the effect of shear stiffness introduced by this model.

The paper is structured as follows. Section 2 presents an overview of the FAST global sensitivity analysis method used in this work. The mathematical framework and sensitivity indices are defined in this section. The general model of acoustic transmission of plane wave and diffuse field through an infinite plate is presented in section 3, then detailed in sections 4, 5 and 6 for the isotropic, orthotropic and sandwich plates respectively. Results of the FAST method are presented and analysed in these sections The case study of a sandwich panel in section 6 enables us to give hints on how this method could be applied to uncertain design in vibroacoustics. 


\section{The FAST method}

\subsection{Analysis of variance}

The influence of a single parameter on the model output can be quantified by the impact it has on the variance in the given design range. In the following development, a generic mathematical model is considered. A model is defined as a real valued function $f$ defined over $K=[0,1]^{n}$. With appropriate variable changes, any function defined over continuous ranges of parameters can be represented that way.

For a given model $f$ mapping a vector of input parameters $\mathbf{x}=\left(x_{1}, \ldots x_{n}\right)$ to a scalar output $y=f(\mathbf{x})$, there exists a unique partition of $f$ so that:

$$
\begin{aligned}
y & =f\left(x_{1}, x_{2}, \ldots x_{n}\right)=\left.\sum_{U \subset[1, n]} f_{U}\left(\left.x\right|_{U}\right) \mathrm{d} x\right|_{U} \\
& =f_{0}+\sum_{i=1}^{n} f_{i}\left(x_{i}\right)+\sum_{1 \leqslant i<j \leqslant n} f_{i j}\left(x_{i}, x_{j}\right)+\ldots+f_{1 \ldots n}\left(x_{1}, \ldots, x_{n}\right),
\end{aligned}
$$

provided that each function $f_{U}$ involved in the decomposition has zero mean over its range of variation $\left.K\right|_{U}$, which is the subspace of $K$ spanned by the dimensions contained in subset $U$. This writes:

$$
\int_{\left.K\right|_{U}} f_{U}\left(x_{U}\right) \mathrm{d} x_{U}=0 .
$$

The decomposition given by Eq. (1) is called the Hoeffding decomposition or high order model representation (HDMR) [13].

For a given set of indices $U=\left\{i_{1}, \ldots, i_{k}\right\}$, the partial variance is therefore the variance of $f_{U}$ :

$$
V_{U}=\int_{\left.K\right|_{U}} f_{U}\left(x_{U}\right)^{2} \mathrm{~d} x_{U}
$$


The sensitivity index relative to the set $U$ is expressed as the ratio of the variance of the function $f_{U}$ to the total variance of the model:

$$
\operatorname{SI}(U)=\frac{V_{U}}{V} .
$$

The computation of all the $2^{n}$ sensitivity indices is needed to fully represent the model, however this quickly becomes a very costly task in terms of computational time, as they have to be evaluated by numerical integration. However, most information about a parameter's influence can be found in the first-order sensitivity index and the total sensitivity index, which can be computed more efficiently with the FAST method.

For a given parameter $i \in[1, n]$, the main effect $(\mathrm{ME})$ is then the sensitivity index relative to the 1-dimensional function $f_{i}$ :

$$
\operatorname{ME}(i)=\operatorname{SI}(\{i\}) .
$$

Another interesting sensitivity measure for a given parameter $i$ is the total sensitivity index, defined as the sum of the indices of all sets of parameters $U$ to which $i$ belongs:

$$
\operatorname{TSI}(i)=\sum_{\substack{U \subset[1, n] \\ i \in U}} \operatorname{SI}(U) .
$$

\subsection{Main effect computation}

The idea of the FAST method is to avoid the evaluation of the multidimensional integrals needed for the computation of the $f_{i}$ functions, and replace them by a single 1-dimensional integral along a space-filling curve in the design space. This curve is defined so as to be periodic with different periods relative to each parameter. Saltelli et al. [19] propose the sampling function defined by: 


$$
x_{i}=\frac{1}{2}+\frac{1}{\pi} \arcsin \left(\sin \left(\omega_{i} s+\varphi_{i}\right)\right) .
$$

The frequencies $\omega_{i}$ are integers chosen so as to minimize interferences between parameters [14], and the $\varphi_{i}$ are random real numbers in the interval $[0,2 \pi]$. The set of integer frequencies $\left\{\omega_{i}\right\}$ is said to be free of interferences up to order $M$ if all linear combinations

$$
\sum_{i=1}^{n} \alpha_{i} \omega_{i} \neq 0
$$

where $\alpha_{i} \in \mathbb{Z}$ and $\sum_{i=1}^{n}\left|\alpha_{i}\right|<M$.

As all the frequencies are integers, the resulting function is $2 \pi$-periodic with respect to variable $s$. A sampling is then done using $N>2 \omega_{n}+1$ samples in the $[0,2 \pi]$ interval. Calling $y_{k}=f\left(x_{k}\right)$ the model output on each sample, the discrete Fourier transform $\hat{y}_{k}$ can be easily computed numerically.

The values of the frequencies $\omega_{i}$ for $M=4$ and less than 19 parameters have been found by Schaibly and Shuler [20] and are recalled up to 6 parameters in Table 1

[Table 1 about here.]

The total variance of the function in the design space is computed with Parseval's theorem as

$$
V=\int_{K}\left(f^{2}(x)-f_{0}^{2}\right) \mathrm{d} x \approx \sum_{k=1}^{N} y_{k}^{2}=\sum_{k=1}^{N} \hat{y}_{k}^{2} .
$$

The contribution of parameter $i$ is then approximated by the sum of the coefficients indexed by the $M$ lowest multiples of $\omega_{i}$ :

$$
V_{i}=\sum_{k=1}^{M} \hat{y}_{k \omega_{i}}^{2}
$$


and the main effect is calculated as

$$
\operatorname{ME}(i)=\frac{V_{i}}{V}
$$

These analysis steps are repeated for several (typically 3) draws of the $\varphi_{i}$ and the results averaged over these draws. This ensures more robustness in the evaluation of the sensitivity indices.

\subsection{Total sensitivity index computation}

A method proposed in [19] is to assign one high frequency $\omega_{i}$ to parameter $i$ and a set of low frequencies $\left\{\omega_{\sim i}\right\}$ to all other parameters. The same sampling curve as defined in Eq. (7) is used with these frequencies. Extracting the low frequency content gives the share of variance due to all parameters but $i$. The total sensitivity index of parameter $i$ is then:

$$
\operatorname{TSI}(i)=1-\frac{V_{\sim i}}{D}
$$

where $V_{\sim i}$ is the partial variance relative to all parameters but $i$.

\subsection{Interpretation}

By definition, the sensitivity indices range between 0 and 1 . The sum of

main effects (ME) is less than or equal to 1 . The difference $1-\sum_{i=1}^{n} \mathrm{ME}(i)$ is a measure of how much interaction there is between parameters to produce the variance, i.e. how much of the variance cannot be explained by variations of each parameter individually. This difference is exactly zero only for functions whose outputs are linear combination of the input parameters, also called additive models. For such models, main effect and total sensitivity index are equal. 
The value of the ME represents the share of the output variance that is explained by the considered parameter alone. Most important parameters therefore have high ME, but a low ME does not mean the parameter has no influence, as it can be involved in interactions.

The total sensitivity index (TSI) is a measure of the share of the variance that is removed from the total variance when the considered parameter is fixed to its reference value. Therefore parameters with low TSI can be considered as non-influential.

\section{Sound transmission through plane structures}

The general set-up that will be studied in the following is made of an infinite plate located in the plane $z=0$, separating two half-spaces filled with a light fluid with density $\rho_{0}$ and characteristic sound speed $c_{0}$. A plane pressure wave of pulsation $\omega$ travels in the half space $z<0$ with an angle $\theta$ with respect to the $x-y$ plane and a trace direction $\phi$ in the $x-y$ plane. The notation is presented in Fig. 1.

[Figure 1 about here.]

This situation leads to a incident acoustic pressure field in the half-space $z<0$ that can be written as:

$$
p_{I}=p_{0} \exp (\mathrm{i}(\omega t-\mathbf{k} \cdot \mathbf{x}))
$$

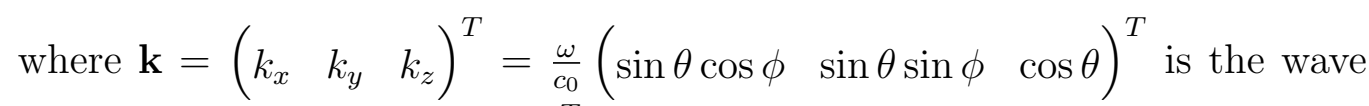
number vector, $\mathbf{x}=\left(\begin{array}{lll}x & y & z\end{array}\right)^{T}$ is the position vector of the current point in space and $\mathrm{i}^{2}=-1$. The sound speed in the fluid is denoted by $c_{0}$. From now 
on, time harmonic dependence of all pressure and displacement quantities will be assumed, and the factor $\exp (\mathrm{i} \omega t)$ will be omitted from the equations in the remaining of the article.

The interaction between the incident wave and the plate creates a forced wave in the plate, with the same pulsation $\omega$. The wave vector of this wave is:

$$
\mathbf{k}=\frac{\omega}{c_{0}} \sin \theta\left(\begin{array}{c}
\cos \phi \\
\sin \phi
\end{array}\right) .
$$

The plate in turn radiates acoustic plane waves on both sides, a transmitted wave of magnitude $p_{T}$ and a reflected wave $p_{R}$ in the $z>0$ and $z<0$ half-spaces respectively.

The coupling is characterized by the continuity of normal speed at the interface between the fluid and the plate, hence on either side of the structure we have :

$$
\frac{\partial p}{\partial z}=\rho_{0} \omega^{2} w
$$

where $w$ is the displacement of a point in the plate in the $z$-direction. On the incident side, we get:

$$
-\mathrm{i} k_{z}\left(p_{I}-p_{R}\right)=\rho_{0} \omega^{2} w
$$

and on the transmission side:

$$
-\mathrm{i} k_{z} p_{T}=\rho_{0} \omega^{2} w
$$

We then have easily:

$$
p_{R}=p_{I}-p_{T}
$$

Introducing the characteristic impedance of the fluid $Z_{0}=\rho_{0} c_{0}$, we obtain:

$$
w=p_{T} \cos \theta /\left(\mathrm{i} \omega Z_{0}\right)
$$


The other equation needed to solve the problem is the forced vibration equation in the plate, which depends on the nature of the plate and the direction of the incident wave. It can be written under the general form:

$$
L\left(k_{x}, k_{y}, \omega\right) w=q,
$$

where $L$ is a linear operator, and the load $q$ is in this case:

$$
q=p_{I}+p_{R}-p_{T}=2\left(p_{I}-p_{T}\right) .
$$

The structural impedance of the system is the ratio between load and normal speed, which reads:

$$
Z=\frac{L}{\mathrm{i} \omega} .
$$

As we are concerned with the forced response of the plate to an oblique plane wave, the projection of the wavenumber onto the plate's plane is constant, and equal to:

$$
k=\frac{\omega}{c_{0}} \sin \theta .
$$

For the cases presented in this paper, the impedance $Z$ can be expressed as a complex number in the frequency domain.

Combining equations 19 through 22, we obtain a relationship between the incident and transmitted pressures:

$$
\frac{p_{T}}{p_{I}}=\left(\frac{Z \cos \theta}{2 Z_{0}}+1\right)^{-1} .
$$

The intensity of sound on one side is $I=\rho c|p|^{2}$, and the acoustic transparency of the plate is defined as the ratio of intensities on both sides, so:

$$
\tau=\frac{I_{T}}{I_{I}}=\left|\frac{p_{T}}{p_{I}}\right|^{2} .
$$


It is usually more convenient to express the noise reduction capability of a structure as a logarithmic quantity, called the transmission loss:

$$
\mathrm{TL}=-10 \log _{10} \tau
$$

It is important to note that this expression for the transmission loss is valid for a plane wave characterized by its direction angles $\theta$ and $\phi$. Another interesting case in the industry is that of the diffuse field, which is a superposition of plane waves from random incidences. This assumption is valid above a limit frequency [2].

The diffuse field transparency can be calculated from the plane wave one as a weighted average over all possible incidence angles $\theta$ and directions $\phi$ :

$$
\tau_{d}=\frac{\int_{\theta_{\mathrm{inf}}}^{\theta_{\mathrm{sup}}} \int_{0}^{2 \pi} \tau(\theta, \phi) \cos \theta \sin \theta \mathrm{d} \phi \mathrm{d} \theta}{\int_{\theta_{\mathrm{inf}}}^{\theta_{\mathrm{sup}}} \int_{0}^{2 \pi} \cos \theta \sin \theta \mathrm{d} \phi \mathrm{d} \theta} .
$$

Several choices can be made concerning the limits of the integration range, we

will consider here a full range with $\theta_{\text {inf }}=0$ (normal incidence) and $\theta_{\text {sup }}=\pi / 2$ (grazing incidence). This choice may have a large influence on the value of the TL, but not on the following sensitivity analysis.

\section{Isotropic plates}

\subsection{Model}

Infinite isotropic plates can be defined by a single geometrical parameter, their thickness $h$, and four material parameters, namely Young's modulus $E$, Poisson's ratio $\nu$, density $\rho$ and hysteretic damping $\eta$. The governing equation for thin isotropic plates is:

$$
D \nabla^{4} w-\omega^{2} m w=q,
$$


where $D=\frac{E h^{3}}{12\left(1-\nu^{2}\right)}(1+\mathrm{i} \eta)$ is the bending stiffness of the plate and $m=\rho h$ is the surface density. As all directions in the plane are equivalent, there is no dependency on the direction $\phi$, so $\nabla=\frac{\partial}{\partial x}=-\mathrm{i} k=-\mathrm{i} \frac{\omega}{c_{0}} \sin \theta$. The equation then reduces to:

$$
\left(D\left(\frac{\omega}{c_{0}}\right)^{4} \sin ^{4} \theta-\omega^{2} m\right) w=q,
$$

and the structural impedance is:

$$
Z(\omega, \theta)=\mathrm{i} \omega m\left(1-\omega^{2} \frac{D}{m c_{0}^{4}} \sin ^{4} \theta\right) .
$$

The acoustic transparency can then be computed as per Eq. (25) for the oblique plane wave case and Eq. (27) for the diffuse field case. In the case of an excitation by a oblique plane wave, the transmission loss exhibits a minimum for the so-called coincidence frequency:

$$
f_{\text {coin }}=\frac{c^{2}}{2 \pi \sin ^{2} \theta} \sqrt{\frac{m}{\operatorname{Re} D}}
$$

where the bending wave group velocity in the plate meets the trace speed of the incident sound wave. At this frequency, the plate's impedance reaches a minimum, which is zero if there is no damping. Bending waves below $f_{\text {coin }}$ are called subcoincident, and supersonic at frequency above coincidence.

\subsection{Results}

A FAST analysis was first conducted on the analytical model of an infinite isotropic plate with $6 \mathrm{~mm}$ thickness, with parameters uniformly distributed in the ranges defined in Table 2, corresponding to typical values for aluminium with $\pm 10 \%$ variation. This rather high thickness has been chosen so that the coincidence frequency is low, around $4 \mathrm{kHz}$ for $\theta=45^{\circ}$. 


\subsubsection{Oblique plane wave}

We first consider the case where the incident sound field is a plane wave with $45^{\circ}$ incidence. The results are presented in Fig. 2. The first-order sensitivity indices (ME) for all four parameters are shown with respect to frequency. It can be seen that they sum up to 1 in the whole range, except in a narrow band around $4 \mathrm{kHz}$, which corresponds to the coincidence frequency.

The density is shown to have a very high ME in low frequencies, while for higher frequencies, Young's modulus is predominant. This is consistent with the classical result that high frequencies are governed by stiffness effects and the low frequencies by mass effects. As the chosen design range is rather narrow, all coincidence frequencies occur rather close to each other.

A high level of interactions between parameters can be observed in the middle of the considered frequency range, where all parameters have rather low ME, but $E$ and $\rho$ exhibit high TSI as can be seen on Fig. 3. This high level of interaction can be explained by the fact that the coincidence frequency is characterized by an important drop in the TL, and that the value of this frequency (see Eq. (31)) is a function of both density and stiffness. It can also be noticed that neither Poisson ratio nor damping have any significant influence in this range. While this was expected for the Poisson ratio, it is more surprising for the damping factor. It means that the location of the coincidence frequency is more important than the actual drop of the TL there, which is controlled by damping.

[Table 2 about here.]

[Figure 2 about here.] 
[Figure 3 about here.]

\subsubsection{Influence of parameter variability range}

The influence of parameter variability range has been studied by varying the ranges of the three parameters $E, \nu$ and $\rho$ without changing that of damping $\eta$, which stays between $0.25 \%$ and $0.75 \%$. These ranges are now set at $\pm 0.1 \%$ of their reference values, as in Table 3 .

[Table 3 about here.]

Fig. 4 presents the results for this case. It can be seen that the discrepancy between ME and TSI no longer exist at coincidence, and the transition between mass-dominated and stiffness dominated is smoother, but the relative influence of parameters stays unchanged compared to the $10 \%$ case, except around coincidence. As damping still varies within $\pm 50 \%$ of its median value, it becomes much more important in the coincidence range. This is what would be expected from a parametric analysis, as the effect of damping is on the TL drop at coincidence. Because of the very low variability of $E$ and $m$, the value of $f_{\text {coin }}$ is practically fixed, and the value of the drop becomes important.

[Figure 4 about here.]

\subsubsection{Diffuse field}

Diffuse field computations in the remaining of this paper are averaged over third-octave bands using 7 points, which are logarithmically spaced in each band. For the $10 \%$ parameter variation case, the diffuse field integration introduces a different behaviour in the higher frequency range. The critical 
frequency is now the minimum of all coincidence frequencies $f_{\text {crit }}=f_{\text {coin }}(\theta=$ $\pi / 2$ ), and lies this time around $2 \mathrm{kHz}$. Below the $f_{\text {crit }}$, mass is still the most influential parameter. However above the critical frequency the most influential parameter is damping, while stiffness (Young's modulus) only has some influence in the bands where the critical frequency occurs most often. This can be explained by the fact that for all frequency $f>f_{\text {crit }}$, there exist one value of angle $\theta$ such that $f=f_{\text {coin }}(\theta)$. In that case, the main effect governing the TL value is effectively the dip at coincidence, and no longer the coincidence frequency itself, so that damping becomes preponderant, even if it still lies in the same range, as can be seen on Fig. 5.

It can also be noted that the density of the plate keeps a small influence in high frequency, due to the fact that grazing incidence, where the mass law is valid up to high frequencies, is always taken into account in the diffuse field calculation.

[Figure 5 about here.]

\section{Orthotropic plates}

\subsection{Model}

An orthotropic plate is characterised by different properties in the $x$ and $y$ directions. The forced wave equation then becomes:

$$
D_{x} \frac{\partial^{4} w}{\partial x^{4}}+2 D_{x y} \frac{\partial^{4} w}{\partial x^{2} \partial y^{2}}+D_{y} \frac{\partial^{4} w}{\partial y^{4}}-m w^{2} w=q,
$$


where the bending stiffness in each axis is:

$$
\begin{aligned}
D_{x} & =\frac{E_{x} h^{3}}{12\left(1-\frac{E_{y}}{E_{x}} \nu_{x y}^{2}\right)}(1+\mathrm{i} \eta) \\
D_{y} & =\frac{E_{y} h^{3}}{12\left(1-\frac{E_{y}}{E_{x}} \nu_{x y}^{2}\right)}(1+\mathrm{i} \eta) \\
D_{x y} & =\frac{h^{3}}{12\left(1-\frac{E_{y}}{E_{x}} \nu_{x y}^{2}\right)}\left(E_{y} \nu_{x y}+2 G_{x y}\right)(1+\mathrm{i} \eta) .
\end{aligned}
$$

Writing $\frac{\partial}{\partial x}=-\mathrm{i} k_{x}=-\mathrm{i} k \cos \phi$ and $\frac{\partial}{\partial y}=-\mathrm{i} k_{y}=-\mathrm{i} k \sin \phi$, the system reduces to the same form as in the isotropic case, with a heading-dependent impedance. The heading dependent bending stiffness is:

$$
D(\phi)=D_{x} \cos ^{4} \phi+2 D_{x y} \sin ^{2} \phi \cos ^{2} \phi+D_{y} \sin ^{4} \phi
$$

and the structure's impedance is then:

$$
Z(\omega, \theta, \phi)=\mathrm{i} \omega m\left(1-\omega^{2} \frac{D(\phi)}{m c_{0}^{4}} \sin ^{4} \theta\right)
$$

The transmission loss is then computed with Eq. (27), taking into account the dependence on heading direction.

\subsection{Unidirectional composite plate}

A unidirectional composite plate is made of very stiff fibres oriented along a given direction, say $x$, held together by a much softer material called the matrix. This results in very different Young's moduli in the longitudinal $(x)$ and transverse $(y)$ directions. The difference can reach two orders of magnitude for some carbon-reinforced composites such as the one presented in first column of Table 4. The chosen variation ranges correspond roughly to $\pm 10 \%$ variation of the reference parameters. 
The main effects of all six parameters on the TL in diffuse field excitation are presented in Fig. 6. The diffuse field transmission loss exhibits the same behaviour that was observed in section 4 with the isotropic plate in the low and high frequency ranges: Mass density is dominant in low frequencies, and damping in high frequencies, for the same reasons. However the orthotropic plate exhibits a range of critical frequencies, between the two corresponding to the $x$ and $y$ directions:

$$
f_{\text {crit }, x}=\frac{c_{0}^{2}}{2 \pi} \sqrt{\frac{m}{\operatorname{Re} D_{x}}} \text { and } f_{c r i t, y}=\frac{c_{0}^{2}}{2 \pi} \sqrt{\frac{m}{\operatorname{Re} D_{y}}} .
$$

For any direction other than the axes, the critical frequency lies between these two, hence the transmission loss is characterized by a minimum zone between these frequencies. The Young's moduli $E_{x}$ and $E_{y}$ are only influential around $f_{\text {crit }, x}$ and $f_{\text {crit }, y}$ respectively. Between these, both damping and mass density are dominant, as the TL is the result of the sum of both subcoincident (masslaw) and supersonic (damping-controlled) waves. Density again has one peak of influence around the highest coincidence frequency. It can be noted that in the considered ranges, neither the Poisson's ratio $\nu_{x y}$ nor the in-plane shear modulus $G_{x y}$ have any practical influence on the transmission loss.

The main effect of all parameters sum up close to 1 , which means that there are practically no interactions between parameters. It is therefore not necessary to compute the TSIs as they would be nearly equal to the main effects.

[Table 4 about here.]

[Figure 6 about here.] 


\subsection{Quasi isotropic plate}

A quasi isotropic plate is obtained by stacking fibre-matrix composite plates with different fibre orientations. They can be modelled as orthotropic plates with equal Young's moduli in $x$ and $y$ directions, but with an independent in-plane shear modulus $G_{x y}$, not equal in general to that of an isotropic plate. In that case, only five parameters will be taken into account, with $E_{x}=E_{y}=E$ and so $D_{x}=D_{y}=D$. The in-plane shear term $D_{x y}$ can be replaced by $\alpha D$, introducing a dimensionless coefficient $\alpha$ as in [21]. It is exactly 1 for isotropic structures, and less than 1 for most types of laminated composites, but can for some constructions be greater than 1 . We chose here arbitrarily to make this parameter vary between 0.4 and 1.2 .

[Table 5 about here.]

Results of the FAST analysis are presented on Fig. 7 in the same fashion than for the unidirectional plate in previous section. The results are close to those of the isotropic plate of section 4 for parameters relevant to both models (damping, density, Poisson's ratio and Young's modulus), and it can be seen that the in-plane shear parameter alpha does not account for much of the TL variance, except in the third octave just above coincidence. This can be explained that, as parameter $\alpha$ is mostly less than 1 on its range of variation, it tends to increase the wavenumber at $45^{\circ}$ of the axes, which leads to a slightly higher coincidence frequency for waves propagating in this direction.

[Figure 7 about here.] 


\section{Uncertain design of a sandwich panel}

\subsection{Model}

A sandwich construction is made of two thin plates, called the skins, glued to a thick core. The core is usually made of very stiff and light material, which ensures a high stiffness-to-mass ratio of the structure, interesting for aerospace applications. However the shear stiffness must be taken into account to accurately model this kind of materials. Out of plane waves propagating in a sandwich plate are no longer purely bending wave, but incorporate some shear deformation form the core. However, other deformations are not considered, which allows to represent the skins with their Young's modulus $E$ and the core by its sole shear modulus $G$. The equation of motion for an isotropic sandwich plate submitted to an external force is given by Renji [22]. Substituting the viscous damping term in the reference by an hysteretic damping, defined as an imaginary part of the bending stiffness $D$, this equation becomes

$$
D k^{4}-\rho \omega^{2}\left(1+\frac{D}{N} k^{2}\right) w=q,
$$

which leads to the following form for the structural impedance:

$$
Z(\omega, \theta)=\mathrm{i} m \omega\left[1+\left(\frac{D}{N c_{0}^{2}} \sin ^{2} \theta-\frac{D}{m c_{0}^{4}}(1+\mathrm{i} \eta) \sin ^{4} \theta\right) \omega^{2}\right]
$$

For sandwiches made of isotropic materials, the bending stiffness is $D=$ $E h_{c}^{2} h_{s}\left(1+\frac{h_{s}}{h_{c}}\right)^{2}$ and the shear stiffness is $N=G h_{c}\left(1+\frac{h_{s}}{h_{c}}\right)^{2}$. This expression is equivalent to that of a thin plate if the shear stiffness is infinite. 


\subsection{Results}

We consider a sandwich panel with four uncertain parameters, whose variations range are summarised in Table 6, namely the sandwich's surface density $m$, the core's shear stiffness $G$, the skin's Young's modulus $E$ and the structural damping $\eta$. The thicknesses of the skins and core were assumed constant, respectively at $h_{s}=1 \mathrm{~mm}$ and $h_{c}=2 \mathrm{~cm}$. These values are in the order of magnitudes attained by sandwich panels made of aluminium skins and honeycomb core. The choice of surface density over material density comes from the fact that the former depends on the properties of the honeycomb core, rather than a homogeneous material, and that this quantity directly arises in the sandwich's constitutive equation (Eq. 37).

[Table 6 about here.]

[Figure 8 about here.]

In high frequencies, the sandwich construction under diffuse field excitation has a very different behaviour from that observed previously with thin plates.

Fig. 8 presents the main effects of each parameter. The main effects plotted do not always sum up to 1 , which indicates interactions between parameters, especially around coincidence, as was observed for the isotropic case studied in section 4 .

The most interesting result is here that the dominant parameter in high frequencies is no longer damping, but the shear modulus $G$, whereas the Young's modulus $E$ is not important in the whole frequency range, except around coincidence, through interactions with the mass and shear modulus. 
This suggests that the variation range set for the shear modulus is very large, and may correspond to a design latitude. If a reduction of the TL in high frequencies is wanted, it is therefore necessary to work out a better range of variation for $G$, while the other parameters' ranges can be kept.

We shall consider the same average value of $G$ (400 MPa here), but with a narrower range of $\pm 50 \mathrm{MPa}$ on the actual value. This remaining uncertainty may be due to the homogenisation of the honeycomb construction of the core. The main effects for this case are presented in Fig. 9. It can be seen that with these values, the most influential parameter in high frequency is now damping, as was observed for the cases studied in the above sections. Shear is only moderately influential throughout the whole frequency range. The standard deviation in the highest frequency range is reduced by $1.5 \mathrm{~dB}$, which is lower than the uncertainty due to the model itself.

[Figure 9 about here.]

Fig. 10 presents the average transmission loss and the standard deviation per octave bands in both cases. The standard deviation is most important around coincidence and in high frequencies. The final case exhibits a reduced standard deviation compared to the initial case, except in the low frequency range where the modified parameter $G$ was not influential. It can be noted that the average TL is slightly lower in the final case.

In this case the FAST method can be used as a tool for both uncertainty quantification and preparation of an optimisation procedure.

[Figure 10 about here.] 


\section{Conclusion}

The FAST method has been applied on several models for sound transmission through infinite plates. It efficiently allows identification of the most influential parameter per frequency range. It is firstly validated with classical results on transmission loss of a plane wave through an isotropic plate: mass is dominant in the lower frequency range as predicted by mass law, while bending stiffness, represented by the Young's modulus, is dominant for high frequencies. Damping only has some influence around coincidence. The case of diffuse field transmission is then studied for isotropic and orthotropic plates. Damping is now found to be dominant in the higher frequency ranges, whereas stiffness is important only around coincidence. Orthotropic plates are characterised by a coincidence zone bounded by two critical frequencies corresponding to the minimum and maximum values of the bending stiffness in the plate's plane.

Finally, the application of the method to an orthotropic sandwich panel illustrates a potential application in vibroacoustic design of structures: used with a rather wide range for the shear modulus of the core, FAST demonstrated a high influence of this parameter in the highest frequency range and around coincidence, where the standard deviation is also maximum, around 3 $\mathrm{dB}$. If a smaller range can be worked out for the variability of this parameter, this influence reduces greatly, and damping becomes preponderant, while the overall standard deviation of the output is significantly reduced.

The FAST sensitivity analysis can therefore be used to identify the most influential parameters, and quantify their effect on the overall variance. In any case, good estimates of parameter variability ranges need to be known 
a priori, as they may greatly influence the results. That being known, the method can be used for both optimisation and parametric uncertainty quantification. The fact that it is a non-intrusive method makes it readily usable with existing models.

\section{Acknowledgements}

The authors would like to gratefully acknowledge Airbus Defence and Space for their financial support. This project is part of the international cooperation project CRIAQ ACOU504_INTL. This work has been performed in cooperation with the COVIA project (French National Research Agency grant number ANR-12-JS09-008-COVIA).

\section{References}

[1] F. J. Fahy, P. Gardonio, Sound and structural vibration, Academic Press, 2007.

[2] C. Lesueur, M. Heckl, J. Delcambre, Rayonnement acoustique des structures, Eyrolles, 1988.

[3] J.-L. Guyader, C. Lesueur, Transmission of reverberant sound through orthotropic viscoelastic multilayered plates, Journal of Sound and Vibration 70 (3) (1980) 319-332.

[4] K. Renji, P. S. Nair, S. Narayanan, Modal density of composite honeycomb sandwich panels, Journal of Sound and Vibration 195 (5) (1996) 687-699. 
[5] R. D'Ippolito, B. Newill, B. Van der Heggen, Uncertainty Modeling for Aircraft Interior Noise - Composites Transmission Loss Optimization, SAE Technical Paper 2013-01-2216.

[6] E. Reynders, Parametric uncertainty quantification of sound insulation values, Journal of the Acoustical Society of America 135 (2014) 19071918.

[7] W. Batko, P. Pawlik, New method of uncertainty evaluation of the sound insuation of partitions, Acta Physica Polonica A 123 (6) (2013) 1012 1015.

[8] R. D'Amico, A. Pratellesi, M. Pierini, N. Baldanzini, Stochastic BEM for the Vibroacoustic Analysis of Three-Dimensional Structures, Advances in Acoustics and Vibration 2011 (2011) article ID 952407, 12 pages.

[9] R. D'Amico, A. Pratellesi, N. Baldanzini, M. Pierini, Reformulation of the Stochastic $\{\mathrm{BEM}\}$ to improve the computational efficiency in the prediction of the vibro-acoustic behaviour of structures with uncertainties, Journal of Sound and Vibration 332 (9) (2013) 2132 - 2148.

[10] A. Cicirello, R. S. Langley, The vibro-acoustic analysis of built-up systems using a hybrid method with parametric and non-parametric uncertainties, Journal of Sound and Vibration 332 (9) (2013) 2165 - 2178.

[11] R. Ohayon, C. Soize, Advanced computational vibroacoustics, Cambridge University Press, 2014.

[12] A. Saltelli, M. Ratto, T. Andres, F. Campolongo, J. Cariboni, D. Gatelli, 
M. Saisana, S. Tarantola, Global Sensitivity Analysis: The Primer, John Wiley \& Sons, 2008.

[13] I. M. Sobol, Sensitivity analysis for nonlinear mathematical models, Mathematical modeling and computational experiment 1 (1993) 407414.

[14] R. I. Cukier, C. M. Fortuin, K. E. Shuler, A. G. Petschek, J. H. Schaibly, Study of the sensitivity of coupled reaction systems to uncertainties on rate coefficients. I. Theory, Journal of Chemical Physics 59 (8) (1973) 3873-3878.

[15] B. Iooss, F. Van Dorpe, N. Devictor, Response surfaces and sensitivity analyses for an environmental model of dose calculations, Reliability Engineering and System Safety 91 (10-11) (2006) 1241-1251, the Fourth International Conference on Sensitivity Analysis of Model Output (SAMO 2004).

[16] M. Ouisse, M. Ichchou, S. Chedly, M. Collet, On the sensitivity analysis of porous material models, Journal of Sound and Vibration 331 (2012) $5292-5308$.

[17] O. Doutres, M. Ouisse, N. Atalla, M. N. Ichchou, Impact of the irregular microgeometry of polyurethane foam on the macroscopic acoustic behavior predicted by a unit-cell model, The Journal of the Acoustical Society of America 136 (4) (2014) 1666 - 1681.

[18] F. G. Leppington, K. H. Heron, E. G. Broadbent, Resonant and non- 
resonant transmission of random noise through complex plates, Proceedings of the Royal Society of London A 458 (2002) 683-704.

[19] A. Saltelli, S. Tarantola, K. P. S. Chan, A quantitative modelindependent method for global sensitivity analysis of model output, Technometrics 41 (1) (1999) 39-56.

[20] J. H. Schaibly, K. E. Shuler, Study of the sensitivity of coupled reaction systems to uncertainties on rate coefficients. II. Applications, Journal of Chemical Physics 59 (8) (1973) 3879-3888.

[21] K. Renji, P. S. Nair, S. Narayanan, Critical and coincidence frequencies of flat panels, Journal of Sound and Vibration 205 (1) (1997) 19-32.

[22] K. Renji, Sound transmission loss of unbounded panels in bending vibration considering transverse shear deformation, Journal of Sound and Vibration 283 (2005) 478-486. 


\section{List of Figures}

1 Notations used for geometric parameters. . . . . . . . . . . 30

2 Main effects for the four parameters for an infinite isotropic plate impinged by a $45^{\circ}$ incident plane wave with $10 \%$ variability on parameters. The effect of $\eta$ is too small to be visible on the figure. . . . . . . . . . . . . . . . . 31

3 Total sensitivity indices of the 4 parameters of an infinite isotropic plate under $45^{\circ}$ incident plane wave with $10 \%$ parameter variability. . . . . . . . . . . . . . . . 32

4 Main effects of the 4 parameters of an infinite isotropic plate under $45^{\circ}$ incident plane wave with $0.1 \%$ parameter variability. 33

5 Main effects for the four parameters of an infinite isotropic plate under diffuse field excitation with $10 \%$ parameter variability. . . . . . . . . . . . . . . . . . . 34

6 Main effects for the six parameters of the unidirectional orthotropic plate. . . . . . . . . . . . . . . . 35

$7 \quad$ Main effects for the five parameters of the quasi isotropic plate 36

8 Main effects of the three parameters of the sandwich plate model with high variability of shear modulus . . . . . . . . . 37

9 Main effects of the four parameters of the sandwich plate model with low variability of shear modulus . . . . . . . . . 38

10 Average value and standard deviations for the two cases of sandwich plates. . . . . . . . . . . . . . . . . 39 


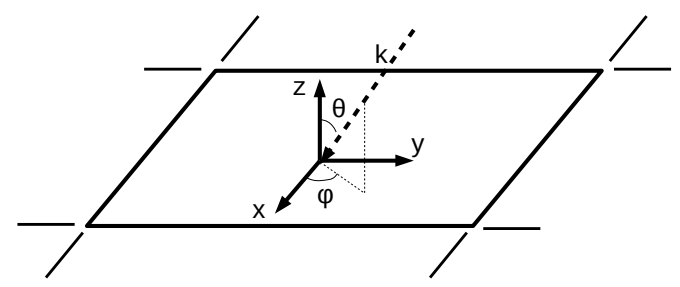

Figure 1: Notations used for geometric parameters. 


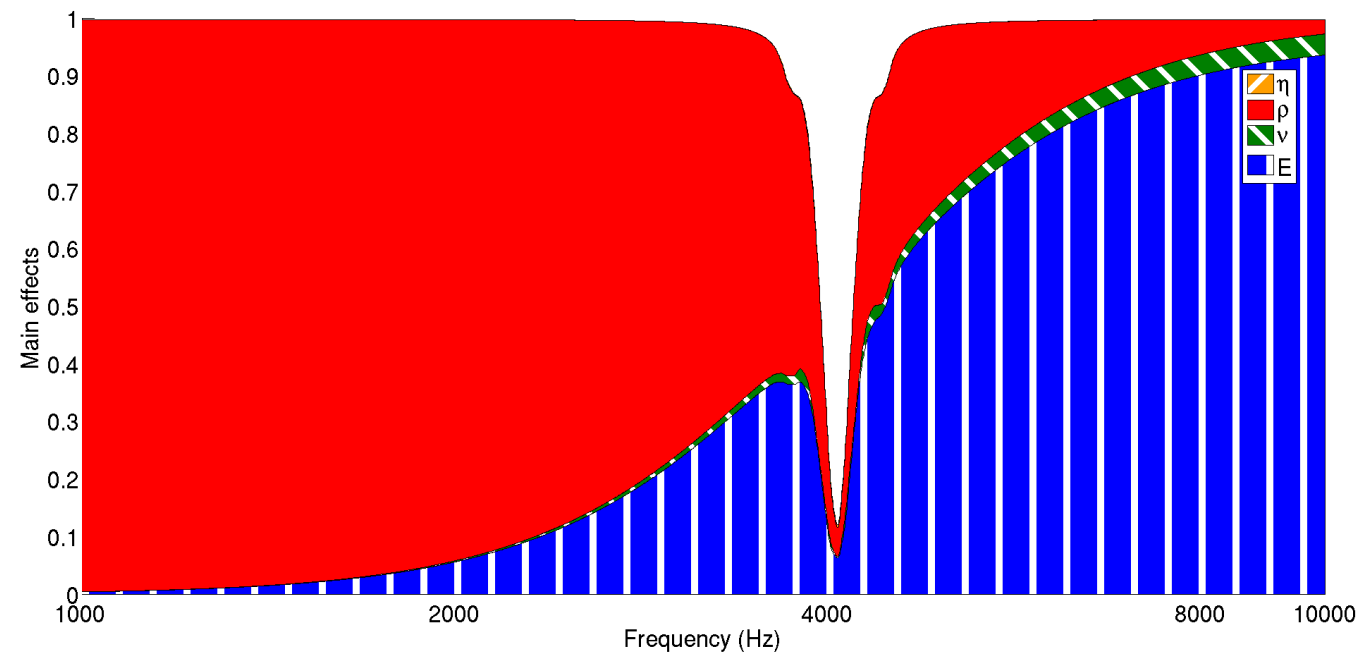

Figure 2: Main effects for the four parameters for an infinite isotropic plate impinged by a $45^{\circ}$ incident plane wave with $10 \%$ variability on parameters. The effect of $\eta$ is too small to be visible on the figure. 


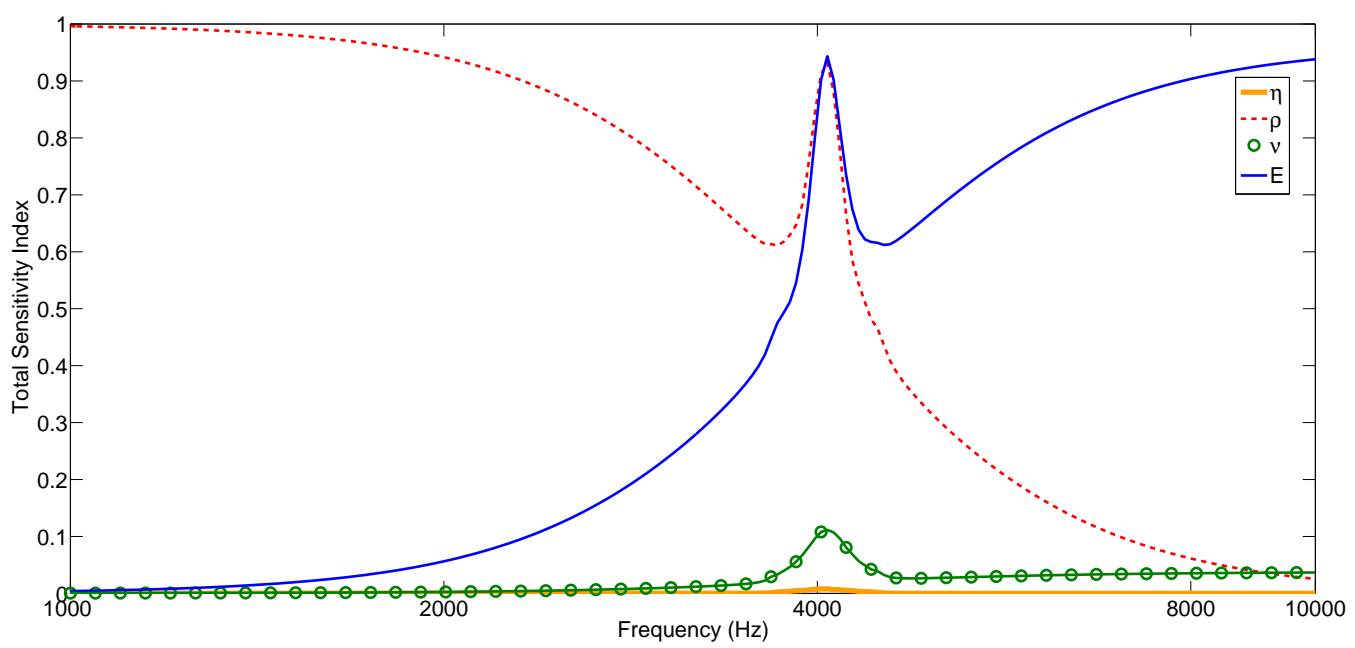

Figure 3: Total sensitivity indices of the 4 parameters of an infinite isotropic plate under $45^{\circ}$ incident plane wave with $10 \%$ parameter variability. 


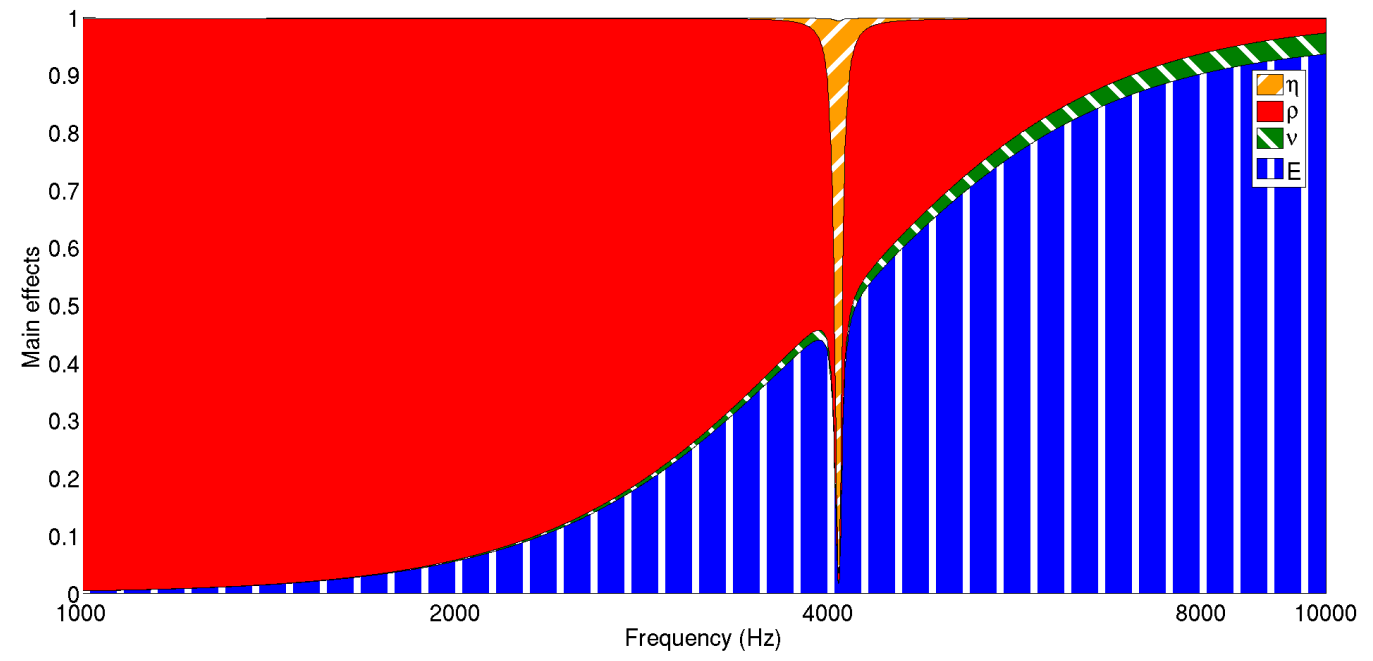

Figure 4: Main effects of the 4 parameters of an infinite isotropic plate under $45^{\circ}$ incident plane wave with $0.1 \%$ parameter variability. 


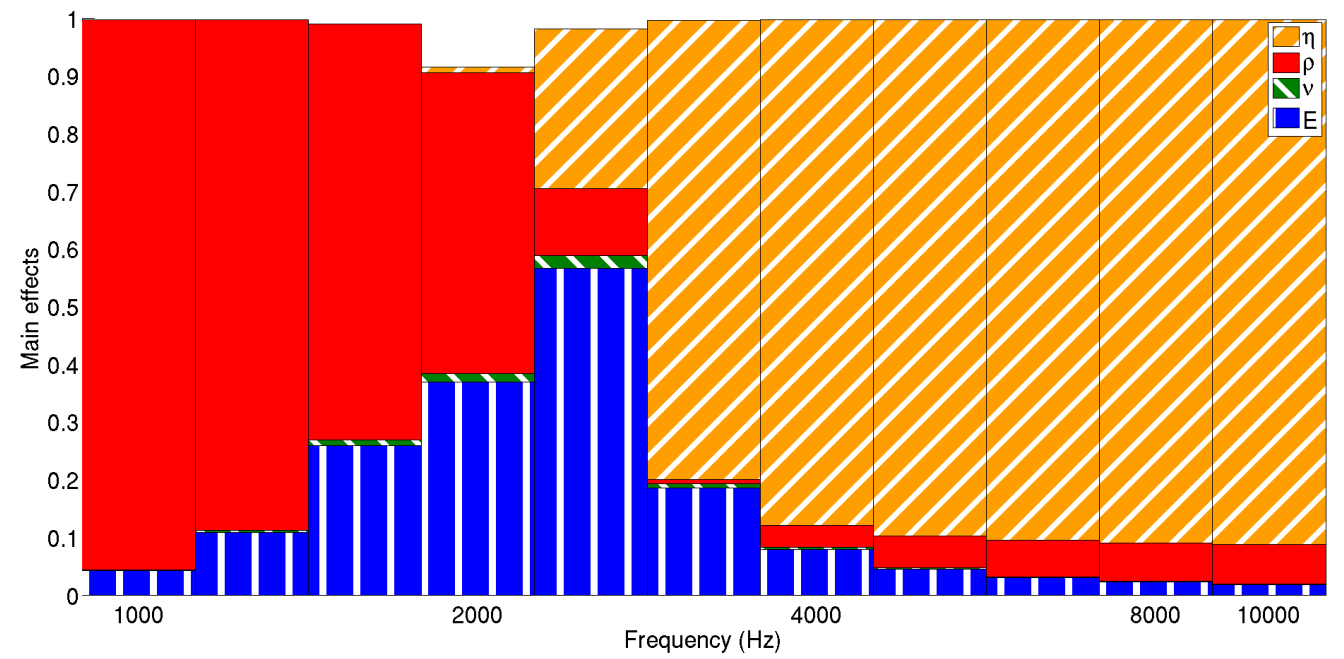

Figure 5: Main effects for the four parameters of an infinite isotropic plate under diffuse field excitation with $10 \%$ parameter variability. 


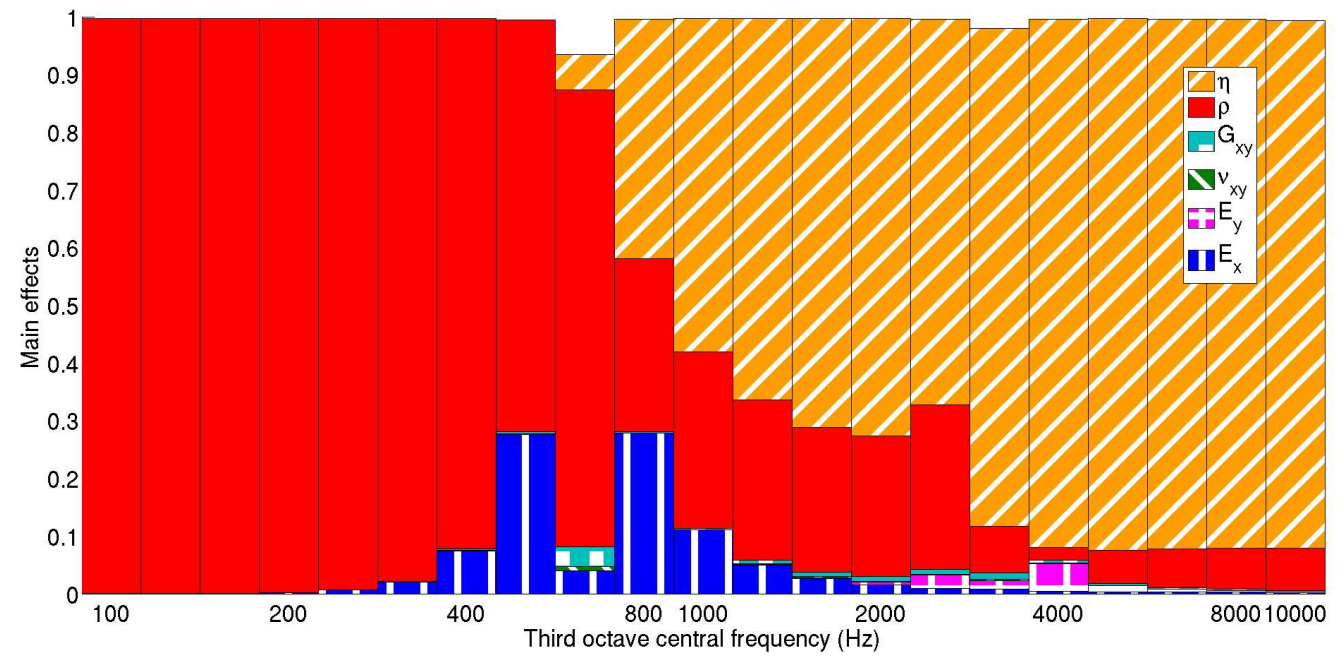

Figure 6: Main effects for the six parameters of the unidirectional orthotropic plate. 


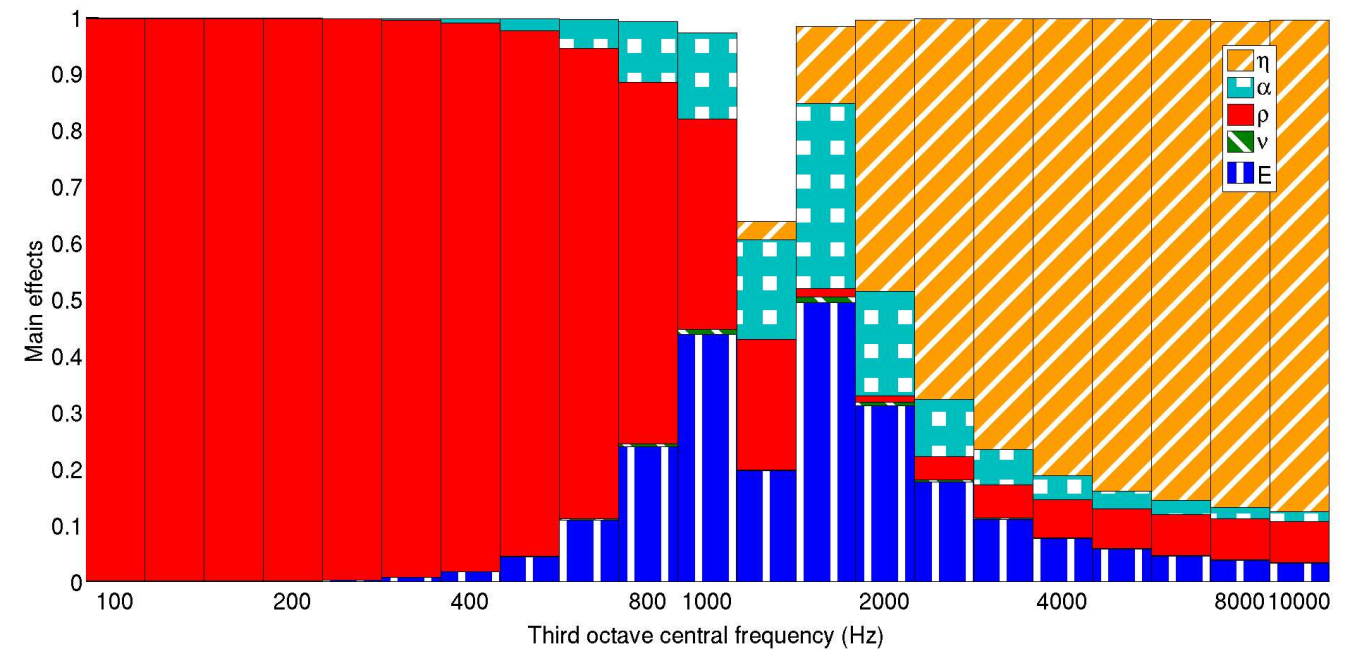

Figure 7: Main effects for the five parameters of the quasi isotropic plate 


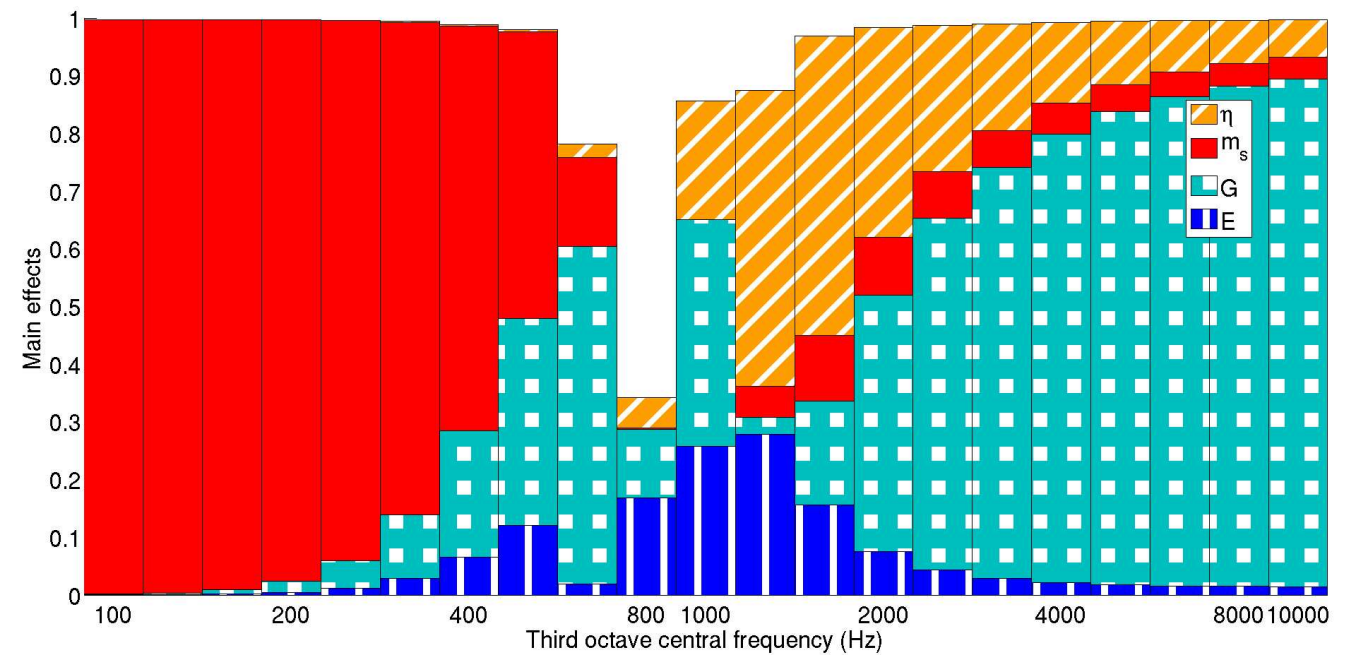

Figure 8: Main effects of the three parameters of the sandwich plate model with high variability of shear modulus 


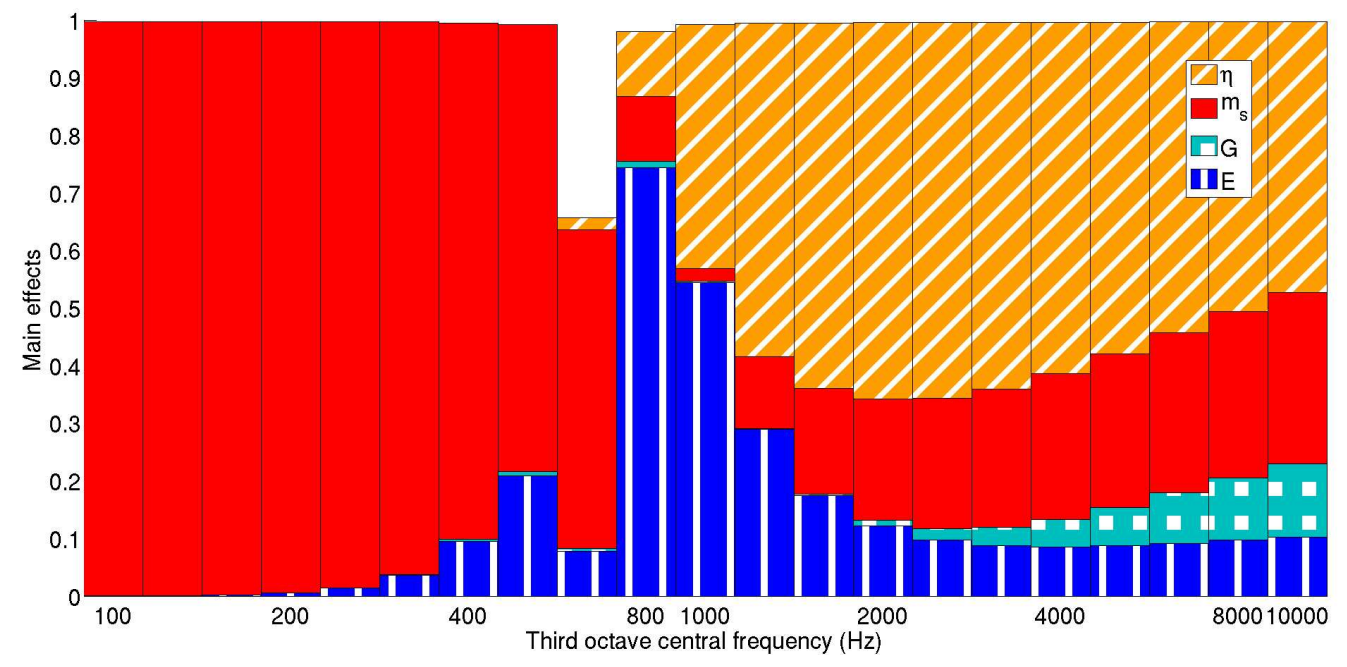

Figure 9: Main effects of the four parameters of the sandwich plate model with low variability of shear modulus 


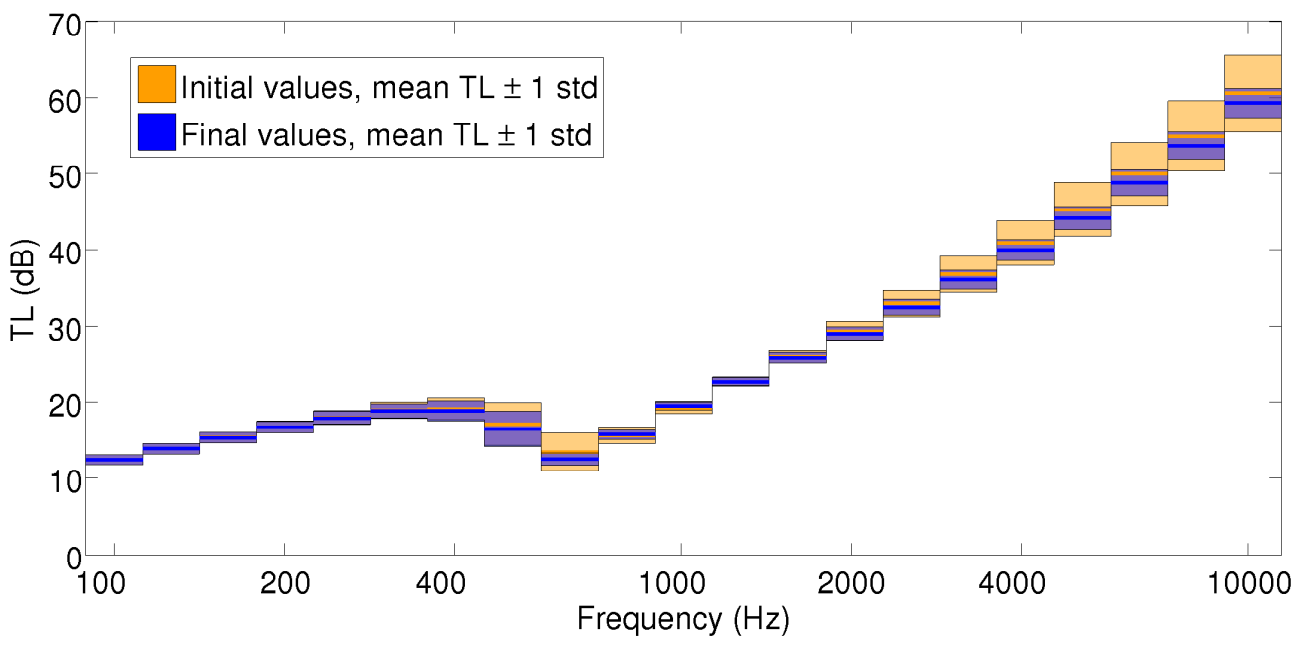

Figure 10: Average value and standard deviations for the two cases of sandwich plates. 


\section{List of Tables}

1 Sets of integer frequencies $\omega_{i}$ for 5 and 6 parameters and $M=$ 4 . For $n<5$, the $n$ smallest values of the set for 5 parameters are used. . . . . . . . . . . . . . . . . . . . 41

2 Variation ranges of the 4 parameters of the isotropic plate model with $10 \%$ variability. . . . . . . . . . . . . . . 42

3 Variation ranges of the 4 parameters of the isotropic plate model with $0.1 \%$ variability . . . . . . . . . . . . . . . 43

4 Values for the FAST analysis of a unidirectional orthotropic plate . . . . . . . . . . . . . . . . 44

$5 \quad$ Values for the FAST analysis of a quasi-isotropic plate . . . . 45

6 Values for the initial FAST analysis of an isotropic sandwich plate . . . . . . . . . . . . . . . . . . 4 46 


\begin{tabular}{cc}
\hline Number of parameters & Set of frequencies \\
\hline 5 & $\{11,21,27,35,39\}$ \\
6 & $\{1,21,31,37,45,49\}$ \\
\hline
\end{tabular}

Table 1: Sets of integer frequencies $\omega_{i}$ for 5 and 6 parameters and $M=4$. For $n<5$, the $n$ smallest values of the set for 5 parameters are used. 


\begin{tabular}{ccc}
\hline Variable & Min. value & Max. value \\
\hline$E_{x}(\mathrm{GPa})$ & 63 & 77 \\
$\nu$ & 0.27 & 0.33 \\
$\rho\left(\mathrm{kg} \cdot \mathrm{m}^{-3}\right)$ & 2430 & 2970 \\
$\eta\left(\cdot 10^{-3}\right)$ & 2.5 & 7.5 \\
\hline
\end{tabular}

Table 2: Variation ranges of the 4 parameters of the isotropic plate model with $10 \%$ variability. 


\begin{tabular}{ccc}
\hline Variable & Min value & Max. value \\
\hline$E_{x}(\mathrm{GPa})$ & 69.93 & 70.07 \\
$\nu$ & 0.2997 & 0.3003 \\
$\rho\left(\mathrm{kg} \cdot \mathrm{m}^{-3}\right)$ & 2697 & 2703 \\
$\eta\left(\cdot 10^{-3}\right)$ & 2.5 & 7.5 \\
\hline
\end{tabular}

Table 3: Variation ranges of the 4 parameters of the isotropic plate model with $0.1 \%$ variability 


\begin{tabular}{ccc}
\hline Variable & Min. value & Max. value \\
\hline$E_{x}(\mathrm{GPa})$ & 201.6 & 246.4 \\
$E_{y}(\mathrm{GPa})$ & 6.21 & 7.59 \\
$\nu_{x y}$ & 0.225 & 0.275 \\
$G_{x y}(\mathrm{GPa})$ & 51.3 & 62.7 \\
$\rho\left(\mathrm{kg} . \mathrm{m}^{-3}\right)$ & 1420 & 1736 \\
$\eta\left(10^{-3}\right)$ & 2.5 & 7.5 \\
\hline
\end{tabular}

Table 4: Values for the FAST analysis of a unidirectional orthotropic plate 


\begin{tabular}{ccc}
\hline Variable & Min. value & Max. value \\
\hline$E_{x}(\mathrm{GPa})$ & 40 & 54 \\
$\nu$ & 0 & 0.2 \\
$\alpha(-)$ & 0.4 & 1.2 \\
$\rho\left(\mathrm{kg} . \mathrm{m}^{-3}\right)$ & 1350 & 1650 \\
$\eta\left(10^{-3}\right)$ & 2.5 & 7.5 \\
\hline
\end{tabular}

Table 5: Values for the FAST analysis of a quasi-isotropic plate 


\begin{tabular}{ccc}
\hline Variable & Min. value & Max. value \\
\hline$E(\mathrm{GPa})$ & 60.0 & 80.0 \\
$G(\mathrm{GPa})$ & 0.1 & 0.7 \\
$m\left(\mathrm{~kg} . \mathrm{m}^{-2}\right)$ & 10 & 14 \\
$\eta\left(10^{-3}\right)$ & 2.5 & 7.5 \\
\hline
\end{tabular}

Table 6: Values for the initial FAST analysis of an isotropic sandwich plate 